\title{
Analysis of Stress in the Periodontal Ligament and Alveolar Bone of the Maxillary First Molars during Intrusion with Microscrew Implants: A 3D Finite Element Study
}

\author{
${ }^{1}$ AS Pavithra, ${ }^{2}$ GS Prashanth, ${ }^{3}$ Silju Mathew, ${ }^{4}$ SE Shekar
}

\begin{abstract}
Objectives: The objective of this study was to graphically display the pattern and magnitude of stress distribution along the periodontal ligament and the alveolar bone of upper first molars on application of intrusive forces using microscrew implants.
\end{abstract}

Materials and methods: A computer simulation of threedimensional model of maxillary first molars and second molars bilaterally with their periodontal ligament and alveolar bone, with microscrew implants, force element and a transpalatal arch were constructed on the basis of average anatomic morphology. Finite element analys is was done to evaluate the amount of stress and its distribution during orthodontic intrusive force.

Results: Overall maximum stress in this study was seen in the alveolar bone in the implant insertion area of $7.155 \mathrm{~N} / \mathrm{mm}^{2}$. Maximum stress in the periodontal ligament was seen in middle third distocervical palatal root surface of the first molar $\left(0.008993 \mathrm{~N} / \mathrm{mm}^{2}\right)$. Maximum stress in the enamel was seen in the distal aspect of the cementoenamel junction $\left(0.423 \mathrm{~N} / \mathrm{mm}^{2}\right)$. Maximum stress in the dentin was observed in apical one-third of the mesiobuccal root surface of first molar $\left(0.1785 \mathrm{~N} / \mathrm{mm}^{2}\right)$.

Conclusion: In this study with the use of palatal implant and transpalatal arch, we found that there was no tipping observed during intrusion. This study demonstrates that significant true intrusion of maxillary molars could be obtained in a wellcontrolled manner by using fixed appliances with microscrew implant as bony anchorage.

Keywords: Microimplant, Molar intrusion, Finite element method, Stress analysis.

How to cite this article: Pavithra AS, Prashanth GS, Mathew $\mathrm{S}$, Shekar SE. Analysis of Stress in the Periodontal Ligament and Alveolar Bone of the Maxillary First Molars during Intrusion with Microscrew Implants: A 3D Finite Element Study. World J Dent 2014;5(1):11-16.

Source of support: Nil

Conflict of interest: None

\footnotetext{
${ }^{1}$ Senior Lecturer, ${ }^{2}$ P rofessor, ${ }^{3,4}$ Professor and Head

${ }^{1}$ Department of Orthodontics, Sri Hasanamba Dental College and Hospital, Hasan, Karnataka, India

2,3 Department of Orthodontics, MS Ramaiah Dental College and Hospital, Bengaluru, Karnataka, India

${ }^{4}$ Department of Orthodontics, KLE Institute of Dental Sciences Bengaluru, Karnataka, India
}

Corresponding Author: AS Pavithra, Senior Lecturer Department of Orthodontics, Sri Hasanamba Dental College and Hospital, Hasan, Karnataka, India, Phone: 8762229832 e-mail: dr.pavithra_3@yahoo.co.in

\section{INTRODUCTION}

The effects of mechanical loads on the tooth-alveolus complex are of particular concern in orthodontics. The mechanical environment associated with orthodontic tooth movement is a unique model in bone adaptation physiology. N umerous finite element models of varying complexity have been developed to calculate tooth movements and stress distributions within the alveolar bone and the periodontal ligament. The stressed state of the periodontal ligament is understood to play a critical role in the tooth movement initiated by orthodontic treatment. A more conservative approach for the over eruption of maxillary molars due to loss of opposing teeth is to intrude the extruded molar orthodontically. ${ }^{1} \mathrm{M}$ icroimplants provide stable intraoral anchorage and enable the maxillary molars to be intruded without the usual side effects.

Lack of well controlled human studies and animal models makes it difficult to achieve an analytical solution for a problem involving complicated geometrics, al though numeric methods, such as finite element, can be used for such studies. The periodontal ligament, tooth and the alveolar bone are all deformable entities under loads. Stress in the periodontal ligament is believed to be the initiating factor in tooth movement, and a range of stresses are transmitted to alveolar bone through the periodontal ligament. Therefore, it is essential to treat these tissues as a continuous unit, so that quantitative assessment of the stress and strain in the periodontium can be done.

The purpose of this study is to simulate the stress response in the periodontal ligament and alveolar bone, of the maxillary first molars to the intrusive force using microscrew implants, since stress in the periodontal ligament is believed to be the initiating factor in tooth movement, and a range of stresses are transmitted to alveolar bone through the periodontal ligament.

\section{MATERIALS AND METHODS}

\section{Construction of the Geometric Model}

The aim was to produce a mathematical model, which represented the biological properties of the tooth and periodontium. In this study, one model of maxillary first 


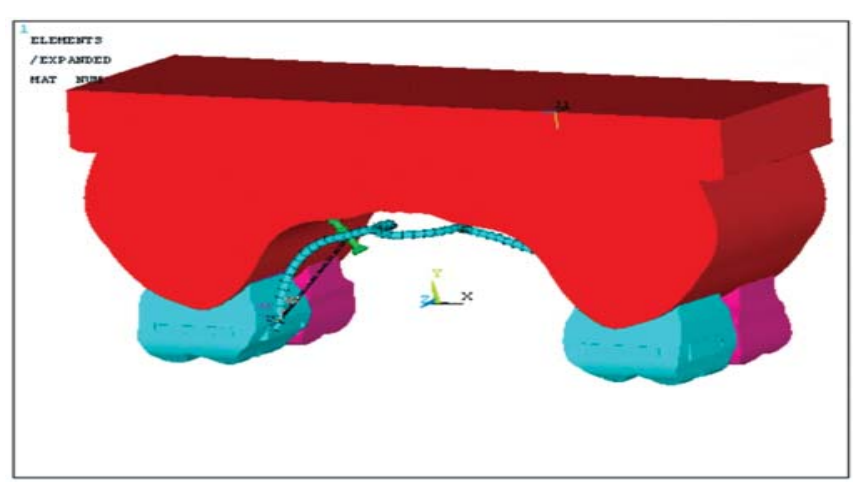

Fig. 1: Initial geometric model of first and second molars with TPA, implant and force element

molars and second molars with their periodontal ligament and alveolar bone, with microscrew implants, force element and a transpalatal bar were constructed. The dimensions in this method were on the based on the data derived from Wheeler's dental anatomy, physiology and occlusion. ${ }^{2} \mathrm{As}$ the thickness of the periodontal ligament is not same all over, an average thickness of $0.28 \mathrm{~mm}$ was assumed and generated around the model of the root. The software used for geometric modelling was A NSY S (version 10) (Fig. 1).

\section{Conversion of the Geometric Model to a Finite Element Method/Discretization}

The continuum is the physical body, structure or solid being analyzed. Discretization may be simply described as the process in which the given body is subdivided into an equivalent system of finite elements. It is achieved by replacing the continuum by a set of key points, called nodes, which when properly connected form the elements. The collection of nodes and elements forms the finite element mesh. The mesh generation constitutes the backbone of the finite element analysis (FEA). The finite elements may be triangles or quadrilaterals for a $2 \mathrm{D}$ continuum. For a $3 \mathrm{D}$ continuum, the finite elements may be either tetrahedral or hexahedral in shape and the elements could be linear quadratic or cubic in order. In this study, to model the irregular geometry of the tooth, tetrahedron meshing was selected as the finite element (Table 1).

\section{Material Property Data Representation}

The different structures involved in this study include tooth, periodontal ligament, alveolar bone, implant, TPA and force element. Each structure has a specific material property. In this study, all the tissues were assumed to be isotropic and elastic (Table 2).

\section{Defining the Boundary Conditions}

The boundary conditions were defined to simulate how the model was constrained and to prevent it from free body

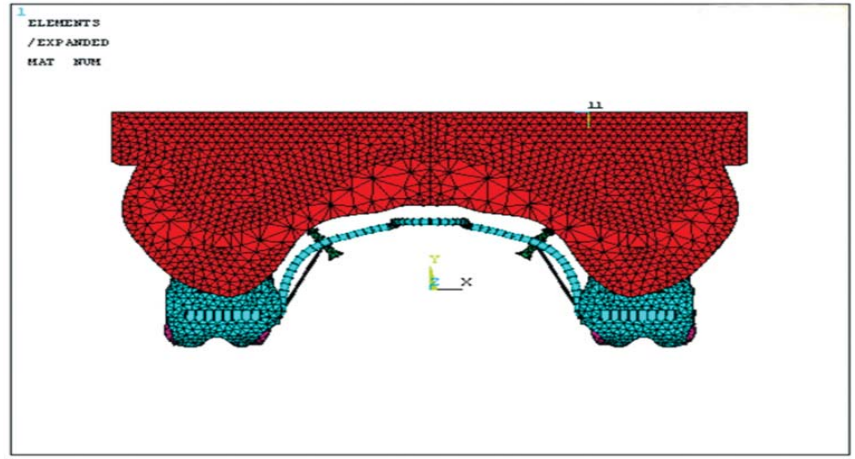

Fig. 2: Three-dimensional mesh model of first and second molars with TPA, implant and force element

motion. The node attached to the area of the top of the bone was fixed.

\section{Application of Forces}

A microscrew implants of diameter $1.2 \mathrm{~mm}$, length of $8 \mathrm{~mm}$, was placed palatally, to $3 \mathrm{~mm}$ thick al veolar cortical bone between maxillary first and second molars on both the sides. The microscrew implants were placed at an angle of $35^{\circ}$ to the long axis of the teeth, with the tip of the screw pointing apically. A transpalatal bar was placed on the upper first molars, to prevent linguoversion of posterior teeth during intrusive force. A $\mathrm{n}$ intrusive force of $150 \mathrm{gm}$ was applied to the maxillary first molars through the force element attached from the palatal implant to the hook of the transpalatal arch (Fig. 2).

\section{Element Stiffness Matrix using a Variation Principle}

The stiffness matrix consists of the coefficients of the equilibrium equations derived from the material and the geometric properties of an element and obtained by the use of principle of minimum potential energy. The stiffness relates the displacements at the nodal points to the applied forces at the nodal points.

The distributed forces applied to the structure were converted into equivalent concentrated forces at the nodes. The equilibrium relation between the stiffness matrix $[K]$, the nodal force vector $\{Q\}$, and the nodal displacement vector $\{q\}$, was expressed as a set of simultaneous linear algebraic equations:

$$
[K]\{q\}=\{Q\}
$$

\section{Assembly of the Algebraic Equations for the Overall Discretized Continuum}

This process includes the assembly of the overall or the global stiffness matrix for the entire body from the individual element stiffness matrices, and the overall global force or load vector from the element nodal force vectors. 
Analysis of Stress in the Periodontal Ligament and Alveolar Bone of the Maxillary First Molars during Intrusion

Table 1: The number of elements and nodes used in the finite element model

\begin{tabular}{lll}
\hline Model & No. of nodes & No. of elements \\
\hline First molar & 5,680 & 25,579 \\
Second molar & 5,650 & 26,020 \\
Periodontal ligament & 7,928 & 29,102 \\
Alveolar bone & 30,863 & $1,56,938$ \\
TPA & 38 & 36 \\
Implant & 1,698 & 6,570 \\
Force element & 57 & 56 \\
\hline
\end{tabular}

Table 2: The material parameters used in the finite element model

\begin{tabular}{lll}
\hline Material & $\begin{array}{l}\text { Young's modulus } \\
\left(\mathrm{N} / \mathrm{mm}^{2}\right)\end{array}$ & Poisson's ratio \\
\hline Tooth & 15,000 & 0.280 \\
Periodontal ligament & 50 & 0.3 \\
Alveolar bone & 10,000 & 0.3 \\
Implant & 65,000 & 0.3 \\
TPA & 65,000 & 0.3 \\
\hline
\end{tabular}

The overall equilibrium relations between the total stiffness matrix $[K]$, the total load vector $\{R\}$ and the nodal displacement vector for the entire body $\{r\}$ will again be expressed as a set of simultaneous equations:

$$
[K]\{r\}=\{R\}
$$

\section{Solutions for the Unknown Displacements}

The algebraic equations assembled are solved for the unknown displacements. In linear equilibrium problems, this is a relatively straightforward application of matrix algebra techniques. How ever, for nonlinear problems, the desired solutions are obtained by a sequence of steps, each step involving a modification of stiffness matrix and or load vector.

\section{Computation of the Element Strains and Stresses from the Nodal Displacements}

The result of an analysis is called post-processing. Stresses $\left(\mathrm{N} / \mathrm{mm}^{2}\right)$ were calculated and presented in colorful bands; different colors represented different stress levels in the deformed state (Fig. 3).

\section{RESULTS}

Stresses were caculated and presented in colorful bands: red color column of spectrum indicates maximum principal stresses and following colors, like orange, yellow, pink, green, blue, represent reducing level of stress, and white color spectrum represents the lowest level of stress.

The results were obtained as displacement of the teeth, periodontal ligament, and alveolar bone and the distribution of stresses in various structures.

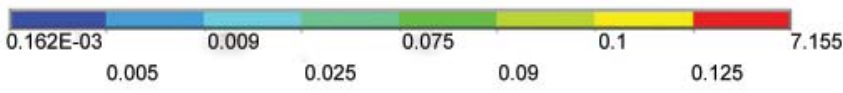

Fig. 3: Results are shown as colorful bands

- Maximum deformation in the alveolar bone was found in the implant area $\left(0.184 \times 10^{-3} \mathrm{~mm}\right)$ and deformation was also seen in mesial and distal cervical alveolar bone of the first molars. Minimum deformation was seen in the palatal alveolar bone (Fig. 4A).

- Maximum stress in the alveolar bone was seen in the implant area $\left(7.155 \mathrm{~N} / \mathrm{mm}^{2}\right)$. M oderate in the mesial and distal cervical alveolar bone (Fig. 4B).

- Displacement in periodontal ligament was maximum at cervical area $\left(0.285 \times 10^{-4} \mathrm{~mm}\right)$ minimum at the mesiobuccal root apex (Fig. 4C).

- Maximum stress in the periodontal ligament was seen in the distal cervical of the palatal root surface of the first molar $\left(0.008993 \mathrm{~N} / \mathrm{mm}^{2}\right)$ and minimum at the palatal root surface of the second molar (Fig. 4D).

- Maximum deformation was seen at the head and neck region of the implant $\left(0.976 \times 10^{-3} \mathrm{~mm}\right)$ and minimal in the tip of the implant (Fig. 4E).

- Maximum stress was seen in the implant at the neck region where it is inserted in the bone $\left(15.258 \mathrm{~N} / \mathrm{mm}^{2}\right)$ and minimum stress was seen in the head region of the implant (Fig. 4F).

- Overall maximum stress in the implant insertion area. M inimum stress was noted in the TPA (Fig. 4G).

- Overall maximum displacement was seen at occlusal one-third of the maillary first molar crown $\left(0.513 \times 10^{-4} \mathrm{~mm}\right)$ and minimum at the root of the second molar (Fig. 4H).

- Maximum stress in the enamel was seen in the distal aspect of the CEJ $\left(0.423 \mathrm{~N} / \mathrm{mm}^{2}\right)$. M inimum stress was seen in the middle one-third of the crown.

- Overall tooth stress was maximum in the distocervical aspect of the first molar $\left(33.981 \mathrm{~N} / \mathrm{mm}^{2}\right)$. M inimum was seen in the palatal root surface of the second molar.

- M aximum stress in the TPA was noted at the joint area to the first molar band $\left(2.298 \mathrm{~N} / \mathrm{mm}^{2}\right)$. M inimum at the Ioop of the TPA.

- M aximum band stress was noted at the distopal atal aspect of the first molar band $\left(0.36196 \mathrm{~N} / \mathrm{mm}^{2}\right)$.

No tipping was observed during intrusion. This study demonstrate that significant true intrusion of maxillary molars could be obtained in a well-controlled manner by using fixed appliances with microscrew implant as bony anchorage.

\section{DISCUSSION}

Finite element method (FEM) provides three-dimensional model with a freedom to simulate orthodontic force system 


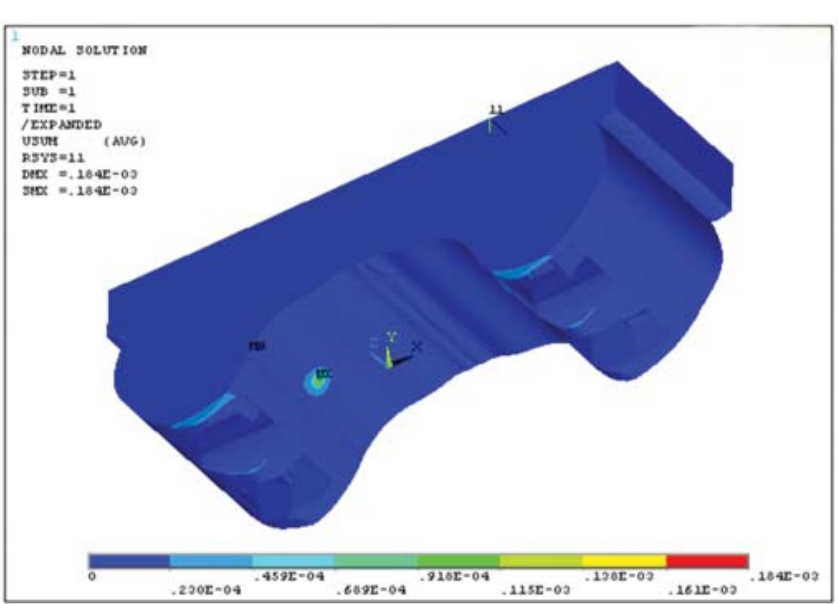

Fig. 4A: Displacement in the alveolar bone when intrusion force was applied to the first molars

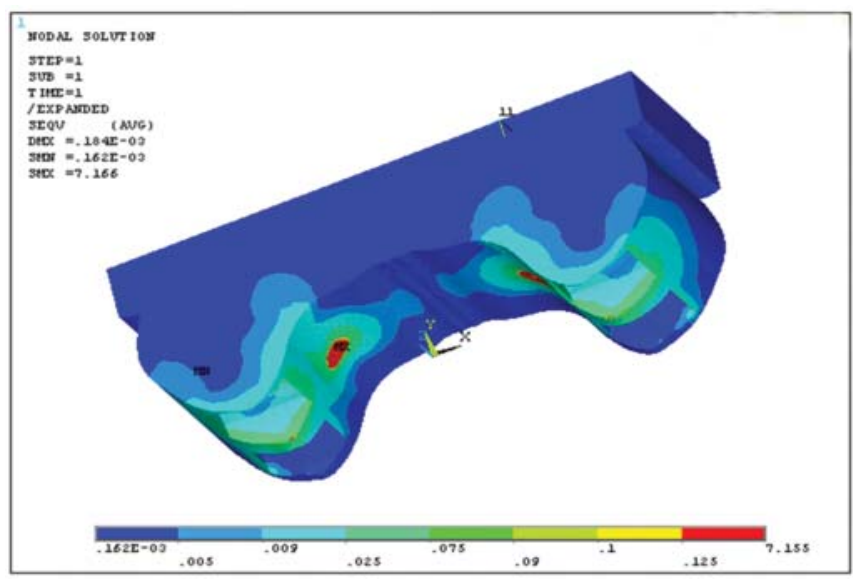

Fig. 4B: Von Mises stress distribution in the alveolar bone when intrusion force was applied to the first molars

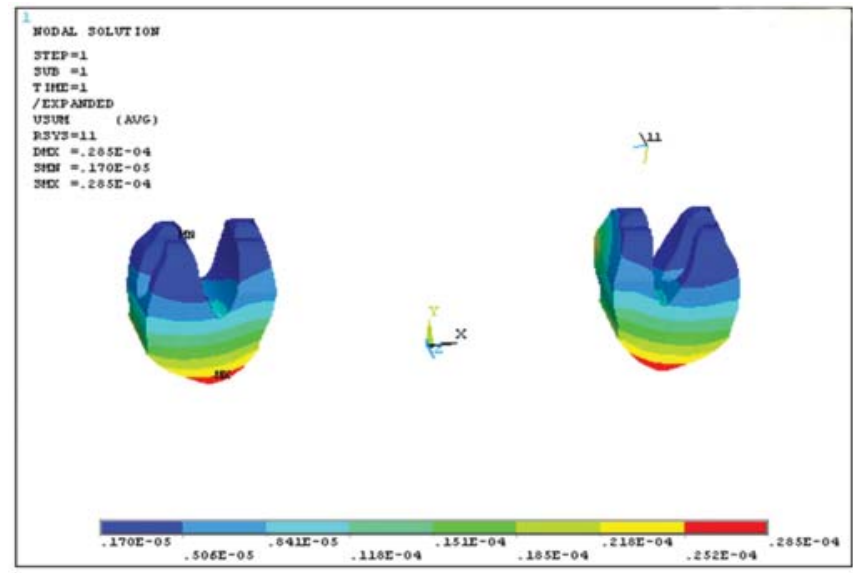

Fig. 4C: Displacement in the periodontal ligament when intrusion force was applied to the first molars

applied clinically and allows the analysis of the reaction of the dentition to orthodontic load in three-dimensional spaces. Finite element analysis makes use of computer to solve large number of equations, which simulates the physical properties of the structure being analyzed. Trying to understand or predict the complexities involved in response to forces has always been a chal lenge for orthodontists.

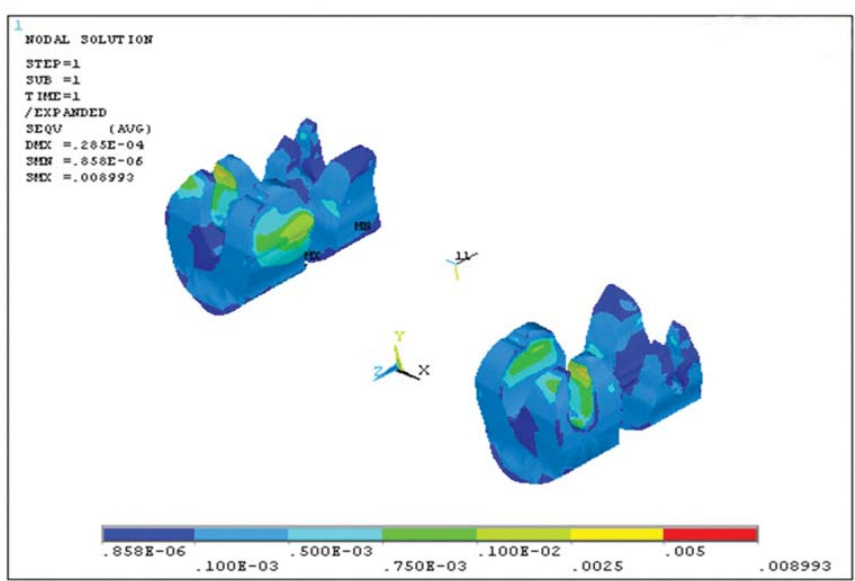

Fig. 4D: Von Mises stress distribution in the periodontal ligament when intrusion force was applied to the first molars

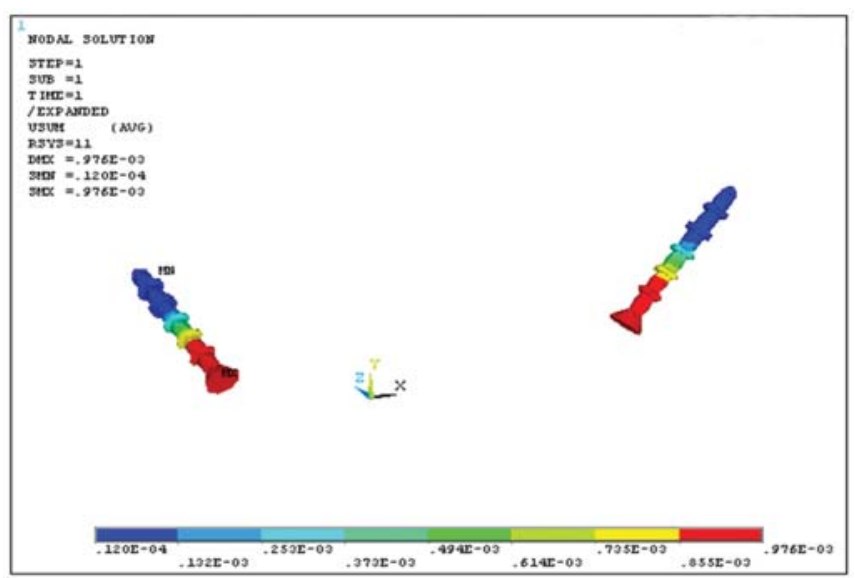

Fig. 4E : Displacement in the implant when intrusion force was applied

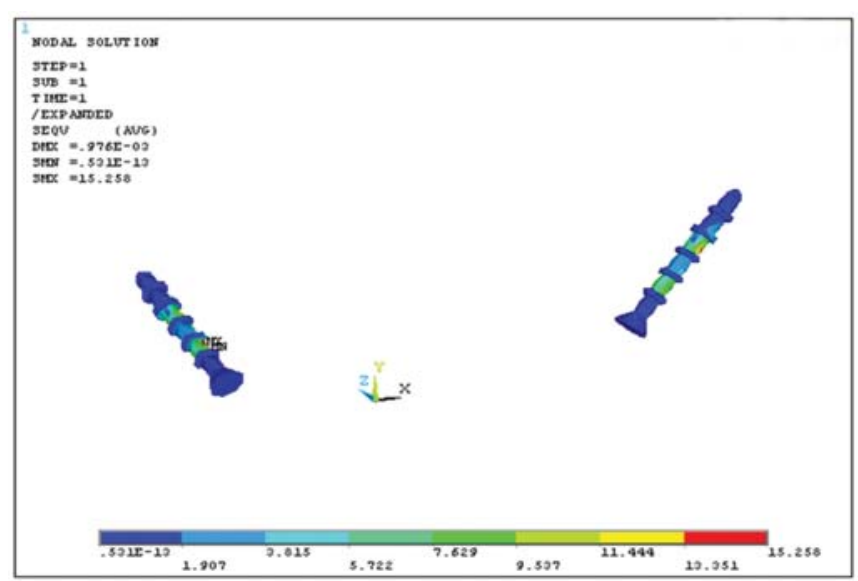

Fig. 4F : Von Mises stress in the implant when intrusion force was applied

A ndersen $\mathrm{KL}$ et al $(1991)^{3}$ conducted a study to evaluate levels and profiles of initial stress in the periodontal ligament after application of various force systems. A s expected, there was a marked variation in stress distribution from cervical area to apex when tipping forces were applied.

Bobak $V$ et al $(1997)^{4}$ carried out finite element method of analysis to analyze theoretically the effects of a 


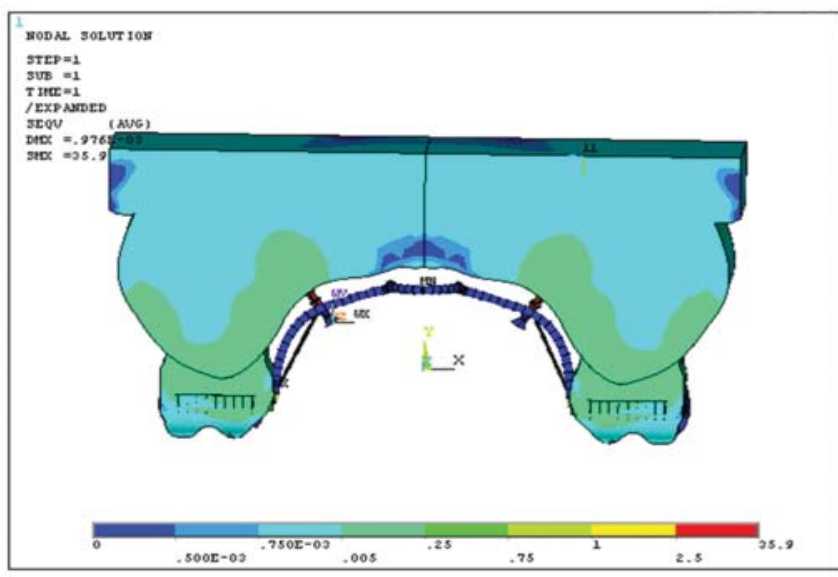

Fig. 4G: Overall Von Mises stress distribution

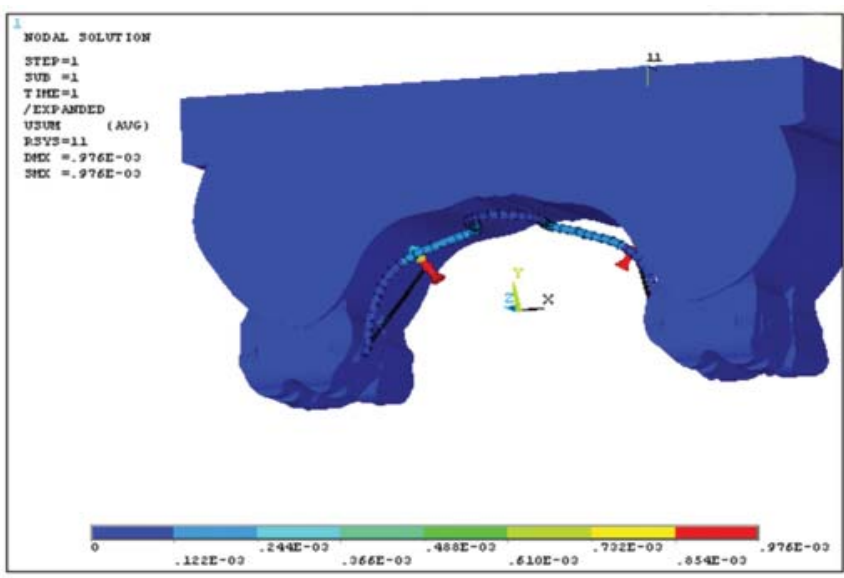

Fig. 4H: Displacement plot of overall structures

transpalatal arch on periodontal stresses of molars that were subjected to typical retraction forces.

Reint Reynders et al $(2009)^{5}$ systematically reviewed and concluded that mini-implants can be used as temporary anchorage devices.

$\mathrm{K}$ Tanne et al ${ }^{6}$ concluded that the Youngs modulus of the PDL was demonstrated to be greater in the adults than in the adolescent subjects. The differing biomechanical properties of the PDL in adults were demonstrated to result in almost equival ent or somew hat increased stress levels in the PDL in adult subjects and thus leads to a delay in tooth movement.

The results given by J eon PD et al (1999) ${ }^{7}$ suggest that the root morphology of the maxillary first molar makes it less susceptible to apical root resorption relative to the anterior teeth during tooth movement. In the current study, when intrusion force of $150 \mathrm{gm}$ was applied to the first molar, maximum stress was observed in apical one-third of the mesiobuccal root surface of first molar $0.178 \mathrm{~N} / \mathrm{mm}^{2}$. A nd, minimum stress in the middle one-third of mesiobuccal root surface of the first molar. Overall maximum stress in this study was found in the implant insertion area in the alveolar bone of $7.155 \mathrm{~N} / \mathrm{mm}^{2}$. It reminded of the initial stress be taken into consideration when immediate loading of the microimplant is planned.

Richard J Smith et al extensively studied the mechanics of tooth movement. They concluded that orthodontic forces can be mathematically treated as vectors, when more than one force is applied to a tooth, the forces can be combined to determine a single overall resultant. ${ }^{8}$

The periodontal tissues exhibit nonlinear behavior in response to external forces. However, the nature of biomechanical behavior of the periodontium before and after orthodontic tooth movement has not been elucidated because of the difficulties in measurement. It is thus important to investigate the biomechanical reactions of the periodontium to the external forces to determine an optimal force application for orthodontic tooth movement. Understanding the principles of biomechanics and the manner in which the tooth and its supporting structures react to forces and moments help to prevent damage to the periodontium during treatment.

Dr R obert M Ricketts said, 'orthodontics is a profession where one enhances the facial esthetics by using the dentition as a tool'. This is even more valid in the 21st century when teeth can be moved much more easily and in a more controlled fashion with miniscrew implants. ${ }^{9}$ One model of maxillary first molars and second molars with their periodontal ligament and alveolar bone, with microscrew implants and a transpalatal bar was developed with defined material properties. A microscrew implants of diameter $1.2 \mathrm{~mm}$, length of $8 \mathrm{~mm}$, was placed palatally, to $3 \mathrm{~mm}$ thick alveolar cortical bone between maxillary first and second molars on both the sides. The microscrew implants were placed at an angle of $35^{\circ}$ to the long axis of the teeth, with the tip of the screw pointing apically. A transpalatal bar was placed on the upper first molars, to prevent linguoversion of posterior teeth during intrusive force. A $n$ intrusive force of $150 \mathrm{gm}$ was applied to the maxillary first molars. The flexibility of the FEM in modifying geometry, allowed the simulation of the stress response in the periodontal ligament, alveolar bone, of the maxillary first molars to the intrusive force using microscrew implants.

In a study conducted by J ohn M iddleton et al in the finite element model analysis, bone was found to experience a low strain of $1 \times 10^{-5} \mathrm{~mm}$, whereas the periodontal ligament experienced a strain of $0.1 \mathrm{~mm}$ when the 'tooth model' is loaded. The results suggest that in this computerized representation of the tissues a strain of significantly greater magnitude is found in the periodontal ligament in comparison to that found in the bone surrounding the tooth. ${ }^{10}$ The current study supports the study by John Middleton et al that the periodontal ligament appears to experience relatively high strains, whereas the adjacent bone appear 
to be in a low strain field. Thus, if strain is accepted as a contributor to the remodelling process, then the findings of this theoretical study support the contention that it is the periodontal ligament that controls the remodelling process rather than the bone itself.

Frost, ${ }^{11}$ after examination of the bone el sewhere in the body, has suggested that for bone remodelling to occur, the strain level should exceed a value of $0.02 \mathrm{~mm}$. There is no reason to expect the situation to be any different for alveolar bone. In the current study, maximum deformation in the alveolar bone was found to be $0.18 \mathrm{~mm}$. Strain in periodontal ligament was maximum at cervical area of $0.28 \mathrm{~mm}$. Thus, arguments put forward by Frost appears to be correct and applicable to dentoalveolus.

The limitations of this study involve approximations in the material behaviors and geometrics of the tissues. The stress-strain relationship of the tissues were assumed linear-elastic and isotropic, and the variation of density and trabeculation of alveolar bone were not considered. The viscous nature of the PDL comes from the tissue fluid, and the elastic behavior from the fibers. The anisotropy results from the orientation of the fibers. Studies have been done to examine anisotropy, ${ }^{12}$ and the viscoelastic behavior ${ }^{13}$ of the PDL which is characterized by time-dependent strain, but these have been limited to two-dimensional analysis. The anisotropic and viscoelastic behavior of the PDL should be examined in further studies using three-dimensional finite element method. In this model, the tooth was simplified as homogenous, and complicated modelling of internal tooth structure was considered unnecessary because force transmission to the PDL is not significantly changed by the internal design of the tooth due to its much high stiffness relative to the PDL. A nother limitation comes from the approximation of tissue geometrics. Variations in root morphology and the PDL thickness may affect stress value and distribution.

\section{CONCLUSION}

This research gains added importance with the increased number of adult orthodontic patients who present themselves for treatment for extruded molars due to early loss of antagonistic tooth.

No tipping was observed during intrusion. This study demonstrate that significant true intrusion of maxillary molars could be obtained in a well-controlled manner by using fixed appliances with microscrew implant as bony anchorage.

\section{REFERENCES}

1. Chun Y S, et al. M axillary molar intrusion with the molar intrusion arch. J ournal of Clinical Orthodontics 2000;34(2):90-93.

2. Nelson SJ, A sh J r M M. W heelers dental anatomy, Physiology and occlusion, 9th ed. Elsevier 2011:171-185.

3. Andersen $K L$, Pedersen EH, Melsen B. Material parameters and stress profiles within the periodontal ligament. A m J Orthod 1991;99:427-440.

4. B obak V, Christiansen RL, H onister SJ , K ohn DH. Stress related molar response to the transpal atal arch: a finite element analysis. A m J Orthod 1997;112:512-518.

5. Reynders R, et al. M ini-implants in orthodontics: A systematic review of the literature. A $m$ J Orthod Dentofacial Orthop 2009;135:564.e1-564.e19.

6. Tanne K, et al. A n evaluation of the biomechanical response of the tooth and periodontium to orthodontic forces in adolescent and adult subjects. B ritish J ournal of O rthodontics 1998;25:109-115.

7. Jeon PD, Turley PK, M oon H, Ting K. A nalysis of stress in the periodontium of the maxillary first molar with a three-dimensional finite element model. A m J Orthod 1999;115:267-274.

8. Smith RJ, Burstone CJ. Mechanics of tooth movement. A merican J ournal of Orthodontics and D entofacial Orthopedics 1984;85:294-307.

9. Paik $\mathrm{CH}$, et al. Orthodontic M iniscrew implants: Clinical applications. Elsevier H eal th Sciences 2009. Introduction chapter Page 5.

10. M iddleton J, et al. The role of the periodontal ligament in bone modelling: the initial development of a time-dependent finite element model. A m J Orhod D entofac Orthop 1996;109:155-162.

11. Frost HM . A determination of bone architecture: the minimum effective strain. Clin Orthop Res 1983; 175:286-292.

12. Beck BW, Harris EF. A pical root resorption in orthodontically treated subjects: analysis of edgewise and light wire mechanics. A m J Orthod Dentofacial Orthop1994;105:350-361.

13. A tmaram $\mathrm{GH}, \mathrm{M}$ ohammed $\mathrm{H}$. Estimation of physiologic stresses with a natural tooth considering fibrous PDL structure. J Dent Res 1981;60:873-877. 